\title{
Gravitational Shock Waves and Their Scattering in Brane-Induced Gravity
}

\author{
Nemanja Kaloper \\ Department of Physics, University of California, Davis, CA 95616
}

(Dated: October 24, 2018)

\begin{abstract}
In this paper, following hep-th/0501028, we present a detailed derivation and discussion of the exact gravitational field solutions for a relativistic particle localized on a tensional brane in braneinduced gravity. Our derivation yields the metrics for both the normal branch and the self-inflating branch Dvali, Gabadadze and Porrati (DGP) braneworlds. They generalize the 4D gravitational shock waves in de Sitter space, and so we compare them to the corresponding 4D General Relativity solution and to the case when gravity resides only in the 5D bulk, and there are no brane-localized graviton kinetic terms. We write down the solutions in terms of two-variable hypergeometric functions and find that at short distances the shock wave profiles look exactly the same as in $4 \mathrm{D}$ Minkowski space, thus recovering the limit one expects if gravity is to be mediated by a metastable, but long-lived, bulk resonance. The corrections far from the source differ from the long distance corrections in $4 \mathrm{D}$ de Sitter space, coming in with odd powers of the distance. We discuss in detail the limiting case on the self-inflating branch when gravity is modified exactly at de Sitter radius, and energy can be lost into the bulk by resonance-like processes. Finally, we consider Planckian scattering on the brane, and find that for a sufficiently small impact parameter it is approximated very closely by the usual $4 \mathrm{D}$ description.
\end{abstract}

PACS numbers: 11.25.-w, 11.25.Mj, 98.80.Cq, 98.80.Qc

hep-th/0502035

\section{INTRODUCTION}

Among many recent braneworld proposals, a particularly interesting idea called brane-induced gravity was put forth by Dvali, Gabadadze and Porrati (DGP) [1]. In this approach the gravitational force between masses on the brane should arise from the exchange of an unstable bulk graviton resonance, which falls apart at very large scales, instead of a stable zero-mode graviton governed by the usual four-dimensional (4D) General Relativity (GR). Far from sources the law of gravity is modified, and changes from the $4 \mathrm{D}$ to a fully higher-dimensional one. This is similar to the approach of [2], which is known to suffer from problems with ghosts [3] (see however [4] for a possible cure of the ghost-induced maladies). The brane-induced gravity setup of DGP may be free of ghosts in perturbation theory [1], and so it is an interesting arena to explore modifications of gravity at large distances. In naive perturbation theory, the modifications appear already at the level of linearized theory, where one finds that the tensor structure of the graviton-matter coupling changes from its form in $4 \mathrm{D}$ GR [1]. Indeed, around flat space, a small perturbation $h_{\mu \nu}$ is sourced by $T_{\mu \nu}-\alpha \eta_{\mu \nu} T_{\lambda}^{\lambda}$, where $\alpha=1 / 3$ instead of $1 / 2$, because of the extra scalar polarization of the massive graviton. Since this coupling does not depend on the graviton mass, the factor $1 / 3$ would appear to persist even as $m_{g} \rightarrow 0$. This is an example of van Dam-Veltman-Zakharov (vDVZ) discontinuity [5], which could modify the predictions for the Solar system tests of general relativity. However, Vainshtein has argued [6] that the naïve perturbation theory for massive gravity breaks down in the limit $m_{g} \rightarrow 0$ because it is a double expansion in both $G_{N}$ and $1 / m_{g}$. Instead, the argument goes, one needs to expand around the curved background created by the source mass, where the curvature generated by the source "screens" the scalar graviton, so that the modified perturbation theory smoothly reduces to conventional GR as $m_{g} \rightarrow 0$. Basically, it is necessary to take into account the non-linear nature of gravity to properly restore gauge invariance which is required to ensure the decoupling of the massive modes as $m_{g} \rightarrow 0$. A similar behavior has also been noticed in non-abelian gauge theories $[\underline{6}$.

The issue of strong coupling in gravity with IR modifications, and in particular its implications for DGP models have been studied in 7, 8, 9, 10, 11, 12. The works 9, 10, 11, 12 specifically address the longitudinal graviton and its matter coupling, and suggest that brane extrinsic curvature may indeed play the role of a coupling controller. When a source mass is placed on the brane, it bends the brane. The brane extrinsic curvature which locally responds to the source may in turn help to tame the perturbation theory 12. This interplay between the source-induced background curvature and the scalar graviton coupling may thus be a key ingredient of the "gravity filter" of [1]. It is therefore essential to explore in detail this interplay of the curvature and the coupling strength beyond perturbation theory in order to clarify the status of the effective 4D theory. However, the DGP equations are very difficult to solve exactly for compact sources localized on the brane [13, 14].

In the previous paper [15] we have presented the first explicit example of an exact solution for a localized particle in DGP models. We have obtained the gravitational field for a relativistic particle on a tensional brane in $5 \mathrm{D}$, which generalizes $4 \mathrm{D}$ gravitational shock waves [16, 17, 18, 19, 20, 21] in de Sitter space [22, 23, 24]. In this paper we follow up with a detailed derivation of the shock wave metrics for both the normal branch and the self-inflating branch variants of DGP braneworlds 
[25]. Focusing on the extreme relativistic limit, we find that the brane-localized terms are indeed a very efficient "gravity filter". At short distances, the wave profile behaves exactly like in 4D GR 17, 18. The deviations appear only far from the source, where they come as odd powers of the distance from the source in the units of the cosmological horizon length, as opposed to the $4 \mathrm{D}$ GR corrections in de Sitter space, which only come as even powers. We also look at gravitational scattering of relativistic particles in DGP braneworlds, and find that to the leading order it behaves the same as in the $4 \mathrm{D}$ case.

We note that our solutions do not provide a direct answer to the conundrum of strong couplings of the scalar graviton. However, we do find that the scalar graviton field is not turned on in these metrics. This would not come as a surprise in a weakly-coupled perturbation theory, where the scalar graviton will not be sourced by ultra-relativistic particles since to the leading order their source vanishes, $T^{\mu}{ }_{\mu}=0$. In other words, in the relativistic limit, one finds that the conformal symmetry is restored, and the solution is form-invariant under boosts. Hence the gravitational shock wave of $4 \mathrm{D}$ GR is also a solution in Brans-Dicke theory. In a more relevant case of very fast particles with non-zero rest mass, the corrections from the mass to the relativistic wave profile should be suppressed by the source mass-to-momentum ratio $m / p \ll 1$. Our results show that decoupling of the scalar graviton persists in the relativistic limit in DGP, and so the dangerous strongly coupled mode identified in perturbation theory does not destroy the solutions at large momenta after all. This suggests that even for the particles with nonzero rest mass the gravitational field may be under control. Indeed, since local physics in DGP obeys the usual $4 \mathrm{D}$ diffeomorphism invariance, we can always pick a very fast-moving observer to explore the field of a massive source, and transform all of the relevant physics to her rest frame. In her frame, thanks to Relativity Principle, the source mass would appear to move with a very high speed $\mathrm{v}$, and therefore its gravitational field should be well approximated by our shock wave solutions. For a sufficiently fast observer, the corrections due to the mass should be suppressed by the powers of $m / p=\sqrt{1 / \mathrm{v}^{2}-1}$. This suggests that if organized as an expansion in powers of $m / p$, perturbation theory may be under control (however, by itself this may not help with any ghost infestations on the self-inflating branch [9]).

The paper is organized as follows. In the next section we construct the background solutions for a tensional brane without particle excitations on it. They are de Sitter vacua of 5D DGP. In section III we present a detailed derivation of the gravitational shock waves, and show that they can be written in terms of two-variable hypergeometric functions. We outline a spectacular new non-perturbative channel for production of bulk gravitons on the self-inflating branch, when gravity is modified at exactly the brane de Sitter radius, in section IV, and consider the limiting form of the solutions at short distances, as well as several special cases in section V.
We then turn to the problem of gravitational scattering of relativistic particles in section VI, and summarize in section VII.

\section{DE SITTER VACUA}

Brane-induced gravity models [1] are given by a bulk action with metric kinetic terms in both the bulk and on the brane. In the case of one extra dimension,

$$
\begin{aligned}
S & =\int d^{5} x \sqrt{g_{5}} \frac{M_{5}^{3}}{2} R_{5}-\int d^{4} x \sqrt{g_{4}} M_{5}^{3} K \\
& +\int d^{4} x \sqrt{g_{4}}\left(\frac{M_{4}^{2}}{2} R_{4}-\lambda-\mathcal{L}_{\text {matter }}\right) .
\end{aligned}
$$

Here $g_{A B}$ and $g_{\mu \nu}=\partial_{\mu} X^{A} \partial_{\nu} X^{B} g_{A B}$ are the $5 \mathrm{D}$ metric and the induced $4 \mathrm{D}$ metric on the brane, respectively, $R_{5}$ and $R_{4}$ are their Ricci tensors, $K=g^{A B} K_{A B}$ is the usual Gibbons-Hawking term necessary in space-times with boundaries (defined as the trace of the extrinsic curvature of the brane, for variational principles see e.g. [26, 27]), and $\lambda$ and $\mathcal{L}_{\text {matter }}$ the brane tension and matter Lagrangian (which we separate from each other explicitly). The indices $\{A, B\}$ and $\{\mu, \nu\}$ are bulk and 3 -brane world-volume indices, and the gravitational couplings are controlled by the bulk and brane Planck scales, $M_{4}$ and $M_{5}$, respectively.

Varying this action away from the brane one finds the gravitational field equations in the bulk, which in the case of empty bulk admit locally 5D Minkowski solution. The brane dynamics can then be incorporated via the modified Israel junction condition [11, 25], which in the case of brane-induced gravity in addition to the brane extrinsic curvature also receives corrections from the intrinsic curvature as well, arising from the variation of $R_{4}$. This junction condition can be viewed as the equation of motion of the brane, defining the world-volume the brane sweeps in the locally Minkowski bulk. In the case of empty bulk, one can readily transform to the rest frame of the brane by going to the Gaussian normal coordinates for the $5 \mathrm{D}$ metric, $d s_{5}^{2}=d w^{2}+g_{\mu \nu}(w, x) d x^{\mu} d x^{\nu}$, and incorporate the brane equations of motion as $\delta$-function terms in the bulk field equations, taking a very compact form. In $5 \mathrm{D}$, those field equations are [1]

$$
M_{5}^{3} G_{5 B}^{A}+M_{4}^{2} G_{4}^{\mu} \delta_{\mu}^{A} \delta_{B}^{\nu} \delta(w)=-T^{\mu}{ }_{\nu} \delta_{\mu}^{A} \delta_{B}^{\nu} \delta(w),
$$

where $G_{5}^{A}{ }_{B}, G_{4 \nu}^{\mu}$ are bulk and 3-brane-localized Einstein tensors, and $T^{\mu}{ }_{\nu}$ is the brane stress-energy, respectively. In this coordinate system one can easily orbifold the bulk, by imposing a $Z_{2}$ symmetry $w \rightarrow-w$ around the brane situated at $w=0$.

For a brane with tension $\lambda \neq 0$ and without any localized or distributed matter sources, $T^{\mu}{ }_{\nu}=-\lambda \delta^{\mu}{ }_{\nu}$. The corresponding solutions describe de Sitter vacua on the brane. The symmetries of the brane-bulk system then dictate the form of the metric, which must be given by 
the warped 4D de Sitter space in a locally flat bulk [25]

$$
d s_{5}^{2}=(1-\epsilon H|w|)^{2} d s_{4 d S}^{2}+d w^{2} .
$$

The replacement $w \rightarrow|w|$ implements the orbifolding procedure. Aside from the parameter $\epsilon$, which can take values \pm 1 in (3), this metric is identical to the $5 \mathrm{D}$ version 28 of the inflating Vilenkin-Ipser-Sikivie domain wall 29]. In what follows we shall use the static patch de Sitter metric,

$$
d s_{4 d S}^{2}=-\left(1-H^{2} r^{2}\right) d t^{2}+\frac{d r^{2}}{\left(1-H^{2} r^{2}\right)}+r^{2} d \Omega_{2},
$$

instead of the spatially flat one utilized in 28]. The space-time of (3) is a $4 \mathrm{D}$ de Sitter hyperboloid in a $5 \mathrm{D}$ Minkowski bulk. Here $\epsilon$ arises because we can retain either the interior $\epsilon=+1$ or the exterior $\epsilon=-1$ of the hyperboloid after orbifolding, thanks to the brane intrinsic curvature $R_{4}$. The junction conditions relate the $4 \mathrm{D}$ curvature and the brane tension as 25]

$$
H^{2}+\epsilon \frac{2 M_{5}^{3}}{M_{4}^{2}} H=\frac{\lambda}{3 M_{4}^{2}} .
$$

The simplest way to derive this equation is to substitute the metric (3) into the field equations (2), recall that away from the brane (3) is just a different parameterization of the locally flat Minkowski metric, and compute the terms proportional to $\delta(w)$, which in this case arise from the double $w$-derivatives in the bulk terms on the LHS in (2), and from the brane curvature terms. Then matching them with the tension source yields (5). We will revisit this derivation in more detail in section III.

Focusing on the solutions with $H>0$ (because $H<0$ cases are their $P T$ transforms), on the normal branch defined by the choice $\epsilon=+1$, we see that the equation (5D) reduces to the $4 \mathrm{D}$ Friedman equation in the limit $M_{5} \ll M_{4}, 3 H^{2} \simeq \lambda / M_{4}^{2}$. In this case in the bulk we keep the interior of the hyperboloid. It has finite volume and so the perturbative $4 \mathrm{D}$ graviton exists. The brane curvature terms $\sim M_{4}^{2} \int d^{4} x \sqrt{g_{4}} R_{4} / 2$ suppress the couplings of the $m_{g}>0$ graviton KK modes, producing the

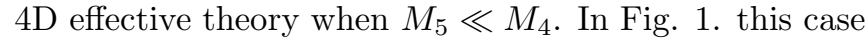
corresponds to keeping the unshaded region of the bulk, i.e. the interior of the de Sitter hyperboloid.

On the other hand, on the self-inflating branch, defined by $\epsilon=-1$, at low tensions $\lambda \ll 12 M_{5}^{6} / M_{4}^{2}$, from the eq. (5I) one finds $H \sim 2 M_{5}^{3} / M_{4}^{2}$. In this case in the bulk one keeps the exterior of the hyperboloid that opens up to infinity, corresponding to the shaded region in Fig. [1 This bulk volume is infinite, and so there is no perturbative $4 \mathrm{D}$ graviton. The effective theory arises only from the exchange of the bulk resonance.

We note that this solution resembles Witten's "bubble of nothing" [30] (see also [31], see Fig. 1), which first collapses from infinite size to a minimum, and bounces back to infinity, gobbling up the bulk along the way. However now the bubble wall carries finite energy density. This may help protect the bulk from being "eaten"

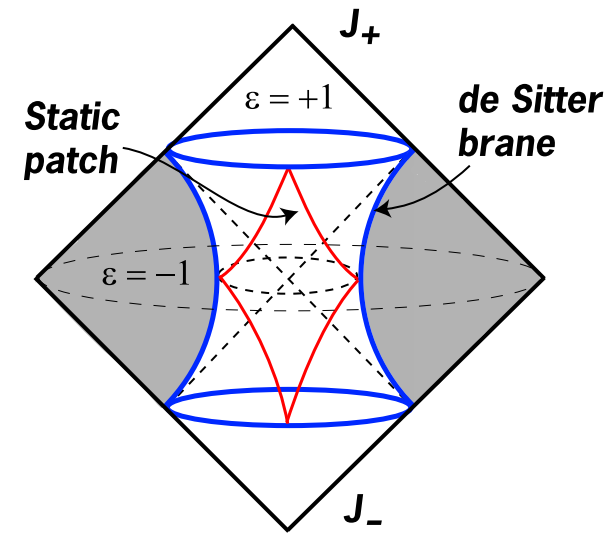

FIG. 1: Penrose diagram of a de Sitter brane in a flat 5D bulk. We suppress one angular coordinate on $S^{2}$ on the brane and depict another, so that the diagram looks like two cones glued along their base, with the hyperboloid of the brane world volume inscribed inside the cones. In the case of the normal branch, in the bulk one retains the interior of the brane worldvolume, denoted with $\epsilon=+1$. For the self-inflating branch, one retains the exterior, marked $\epsilon=-1$. The static patch on the brane is the interior of the diamond-shaped region on the brane world-volume.

by "nothing" too quickly. Namely, the probability of nucleating a "bubble of nothing" is given by the exponential of the negative action of its Euclidean extension, $\mathcal{P} \simeq \exp \left(-S_{E}\right)$. If we take the self-inflating branch solutions (3) and glue them to a Euclidean hemisphere at the minimum radius to model bubble nucleation, we find that the corresponding action is

$$
S_{E}=\frac{V_{4}}{2}\left(\lambda+16 M_{5}^{3} H\right)
$$

where $V_{4}$ is the volume of the sphere of radius $H^{-1}$ (for a simple calculation of the action with Gibbons-Hawking terms see e.g. [26, 32]). In the limit $\lambda \ll 12 M_{5}^{6} / M_{4}^{2}$, $M_{5} \ll M_{4}$, only big bubbles with a large Euclidean action, $S_{E} \sim\left(M_{4} / M_{5}\right)^{6} \gg 1$, are nucleated. Therefore, if the tension and the bulk Planck scale are somehow fixed to be much smaller than the $4 \mathrm{D}$ Planck scale $M_{4}$ (see e.g. [33] for the discussion of scales in brane-induced gravity) the nucleation rate of "bubbles of nothing" may be sufficiently slow to render the self-inflating branch long-lived.

\section{DERIVATION OF SHOCK WAVES}

We now turn to the construction of the gravitational shock wave fields generated by relativistic particles moving on the brane in DGP [15]. Imagine that a photon is moving on a tensional brane, with a momentum $p=2 \pi \nu$. Because it is a relativistic particle trapped on the brane, it moves along a null geodesic of the braneinduced metric. By the Principle of Equivalence, its momentum generates gravitational field, since it contributes 
to the stress-energy tensor. There are two approaches for generating the gravitational field solutions. One could start with a linearized solution for a massive particle at rest, and boost it to relativistic speed, simultaneously taking the limit $m \rightarrow 0$ such that $m \cosh \gamma=p$ remains constant. This is how the solutions in $4 \mathrm{D}$ were originally found [16, 17]. The procedure is completely analogous to the method of generating the electro-magnetic potential of a charged massless particle (for a nice review, see [21]). The same procedure has also been applied to the massless particles moving in maximally symmetric spaces 22, 23. and Randall-Sundrum braneworld models [34, 35]. The reason why this procedure works as a method of generating exact solutions of gravitational field equations is that if we expand the exact metric for a massive source in powers of the mass $m$, after a boost to relativistic speeds the quadratic and higher powers of $m$ vanish because there is only one factor of the boost parameter $\cosh \gamma$ in the metric, which is compensated by one power of $m$ when $\cosh \gamma \rightarrow \infty, m \rightarrow 0$ and $m \cosh \gamma=p=$ const. Hence the linearized solution becomes exact. Essentially, in the extreme relativistic limit the solution becomes scale invariant, and the non-linear effects which are controlled by the fixed ratio $m / M_{4}$ drop out.

The previous discussion on boosting linearized solutions describing point masses to generate exact solutions of gravitational field equations illuminates the nature of the solution as a planar shock wave. Because of the infinite boosting the field lines of the gravitational field become confined to a plane perpendicular to the direction of motion. In terms of the null coordinate $u$ which parameterizes the world-line of the particle $v$, the field experiences a jump at $u=0$ encoding causal propagation of the source. When the particle flies by an observer, its gravitational field perturbs the observer at precisely that instant [18]. This property allowed Dray and 't Hooft to develop a very elegant cut-and-paste technique [18] to find solutions, which they applied to asymptotically flat 4D backgrounds. Higherdimensional examples were considered in [19, 20]. This technique was later applied to general $4 \mathrm{D}$ GR backgrounds by Sfetsos 24]. To circumvent the need for a detailed form of the linearized solution for a localized mass in DGP, we will apply a variant of this cut-and-paste technique to DGP. We start by rewriting (31) in suitable null coordinates, defining $u=\frac{1}{H} \sqrt{\frac{1-H r}{1+H r}} \exp (H t)$ and $v=\frac{1}{H} \sqrt{\frac{1-H r}{1+H r}} \exp (-H t)$. We also introduce a new bulk coordinate $|z|=-\frac{1}{\epsilon H} \ln (1-\epsilon H|w|)$. It simplifies evaluating the curvature tensors with a conformal map as in [36]. Note that $\delta(w)=\delta(z)$ because the warp factor and its derivative are equal to unity on the brane. In terms of these coordinates, we can rewrite the metric (3) in the following form:

$$
d s_{5}^{2}=e^{-2 \epsilon H|z|}\left\{\frac{4 d u d v}{\left(1+H^{2} u v\right)^{2}}+\left(\frac{1-H^{2} u v}{1+H^{2} u v}\right)^{2} \frac{d \Omega_{2}}{H^{2}}+d z^{2}\right\} .
$$

Suppose now that a photon moves along a null geodesic on the brane, let's say the $v$-axis of the coordinates utilized in (7). The equation of this null geodesic is $u=0$. If we choose the origin of this photon at infinite past as the North Pole of the static patch, an observer located there sees a photon streaming away along the past horizon. Following Dray and 't Hooft, we introduce the shock wave on top of the background (77) by including a jump in the $v$ coordinate at $u=0$ [18]. We substitute

$$
\begin{aligned}
& v \rightarrow v+\Theta(u) f, \\
& d v \rightarrow d v+\Theta(u) d f,
\end{aligned}
$$

where $f$ is the shock wave profile, whose precise form is to be determined by requiring that combining (7) and (8) still remains a solution of the field equations. The wave profile $f$ depends only on the spatial coordinates transverse to the wave, which in our case are the angles on the 2-sphere and the bulk coordinate $z$. Here $\Theta(u)$ is the Heaviside step function. Note that in flat $4 \mathrm{D}$ background (8) is a trivial diffeomorphism if $f=$ const. [18]. In that surroundings, if $d f \neq 0$, the replacement rule for $d v$ in (8) ensures this is not just a diffeomorphism. Changing the coordinates to $\hat{v}=v+\Theta(u) f$ we find $d v \rightarrow d \hat{v}-\delta(u) f d u$. Substituting $v, d v \rightarrow \hat{v}, d \hat{v}-\delta(u) f d u$ in (7) and dropping the carets, we obtain the ansätz for the metric with the shock wave included:

$$
\begin{aligned}
d s_{5}^{2}=e^{-2 \epsilon H|z|}\{ & \frac{4 d u d v}{\left(1+H^{2} u v\right)^{2}}-\frac{4 \delta(u) f d u^{2}}{\left(1+H^{2} u v\right)^{2}}+ \\
& \left.+\left(\frac{1-H^{2} u v}{1+H^{2} u v}\right)^{2} \frac{d \Omega_{2}}{H^{2}}+d z^{2}\right\} .
\end{aligned}
$$

Note that with this, the ansätz for the induced metric on the brane is

$$
\begin{array}{r}
d s_{4}^{2}=\frac{4 d u d v}{\left(1+H^{2} u v\right)^{2}}-\frac{4 \delta(u) f d u^{2}}{\left(1+H^{2} u v\right)^{2}}+ \\
+\left(\frac{1-H^{2} u v}{1+H^{2} u v}\right)^{2} \frac{d \Omega_{2}}{H^{2}} .
\end{array}
$$

Now we take the ansätz (9), (10) and substitute it into the field equations (2), where we also include the photon stress-energy contribution in the source on the RHS. Thus the total stress-energy tensor with a photon moving along $u=0$ is

$$
T_{\nu}^{\mu}=-\lambda \delta^{\mu}{ }_{\nu}+2 \frac{p}{\sqrt{g_{5}}} g_{4 u v} \delta(\theta) \delta(\phi) \delta(u) \delta_{v}^{\mu} \delta_{\nu}^{u} .
$$

We have chosen the coordinates on the 2 -sphere such that the photon trajectory is at $\theta=0$.

To evaluate the curvature we utilize the conformal transformation trick as in [36], splitting the metric as $d s_{5}^{2}=\Omega^{2} d \bar{s}_{5}^{2}$, where $\Omega=\exp (-\epsilon H|z|)$, and

$$
\begin{array}{r}
d \bar{s}_{5}^{2}=\frac{4 d u d v}{\left(1+H^{2} u v\right)^{2}}-\frac{4 \delta(u) f d u^{2}}{\left(1+H^{2} u v\right)^{2}}+ \\
+\left(\frac{1-H^{2} u v}{1+H^{2} u v}\right)^{2} \frac{d \Omega_{2}}{H^{2}}+d z^{2} .
\end{array}
$$


A straightforward albeit tedious computation, where we treat $\delta(u)$ and its derivatives as distributions, and use the distributional identities $u \delta(u)=0, u^{2} \delta^{2}(u)=0$ and $f(u) \delta^{\prime}(u)=-f^{\prime}(u) \delta(u)$ [18, 24], yields the Ricci tensor for the conformal metric (12). The stress-energy (11) is automatically conserved, $\nabla_{\mu} T^{\mu}{ }_{\nu} \propto u \delta(u)=0$, so the matter sector field equations are satisfied. We note that this computation also yields automatically the components of the Ricci tensor of the induced metric on the brane, which appear as the subset of the curvature tensor for the conformal metric (12), because $\Omega(0)=1$ and the coordinates in (9) are Gaussian, as is obvious from comparing (10) and (12). The resulting non-vanishing Ricci tensor components are

$$
\begin{aligned}
& \bar{R}_{5 u u}=2 \delta(u)\left(\partial_{z}^{2} f+H^{2}\left(\Delta_{2} f-4 f\right)\right), \\
& R_{4 u u}=2 H^{2} \delta(u)\left(\Delta_{2} f-4 f\right), \\
& \bar{R}_{5 u v}=R_{4 u v}=\frac{6 H^{2}}{\left(1+H^{2} u v\right)^{2}}, \\
& \bar{R}_{5 a b}=R_{4 a b}=3\left(\frac{1-H^{2} u v}{1+H^{2} u v}\right)^{2} g_{a b},
\end{aligned}
$$

where $\bar{R}_{5 A B}$ and $R_{4 \mu \nu}$ are the Ricci tensors of the metrics (12) and (10), and $a, b$ and $g_{a b}$ are the indices and the metric on the unit 2 -sphere, respectively. The operator $\Delta_{2}$ is the Laplacian on the unit 2-sphere.

Using the conformal transformation $d s_{5}^{2}=\Omega^{2} d \bar{s}_{5}^{2}$ which gives the relationship

$$
\begin{aligned}
R_{5 A B}=\bar{R}_{5 A B} & -3 \bar{\nabla}_{A} \bar{\nabla}_{B} \ln \Omega+3 \bar{\nabla}_{A} \ln \Omega \bar{\nabla}_{B} \Omega \\
& -\bar{g}_{A B}\left(\bar{\nabla}^{2} \ln \Omega+3(\bar{\nabla} \ln \Omega)^{2}\right),
\end{aligned}
$$

we finally find

$$
\begin{aligned}
R_{5 z z}= & 8 \epsilon H \delta(z) \\
R_{5 u u}= & 2 \delta(u)\left(\partial_{z}^{2} f-3 \epsilon H \partial_{|z|} f+H^{2}\left(\Delta_{2} f+2 f\right)\right) \\
& -8 \epsilon H f \delta(u) \delta(z) \\
R_{5 u v}= & \frac{4 \epsilon H}{\left(1+H^{2} u v\right)^{2}} \delta(z) \\
R_{5 a b}= & \frac{2 \epsilon}{H}\left(\frac{1-H^{2} u v}{1+H^{2} u v}\right)^{2} \delta(z) g_{a b} .
\end{aligned}
$$

Although Ricci tensor vanishes away from the brane at $z=0$ even when the shock wave is present $\left(R_{5}\right.$ uu in (15) vanishes at $z \neq 0$ by virtue of the field equations, see below), the bulk is not Minkowski any more, since the waves extend off the brane and deform the bulk. One would find their non-vanishing contributions away from the brane in the Riemann tensor. Now from (13) and (15) we can obtain the explicit form of the field equations (2). First, it is easy to check that we can rewrite (2) as

$$
\begin{aligned}
& M_{5}^{3} R_{5 z z}+\frac{M_{4}^{2}}{3} R_{4} \delta(z)=\frac{4}{3} \lambda \delta(z) \\
& M_{5}^{3} R_{5 \mu \nu}+M_{4}^{2}\left(R_{4 \mu \nu}-\frac{1}{6} g_{4 \mu \nu} R_{4}\right) \delta(z)= \\
& \quad=\frac{\lambda}{3} g_{4 \mu \nu} \delta(z)+H^{2} p\left(g_{4 u v}\right)^{2} \delta(\Omega) \delta(u) \delta(z) \delta_{\mu}^{u} \delta_{\nu}^{u} .
\end{aligned}
$$

Here $\delta(\Omega)$ is the covariant $\delta$-function on the unit 2-sphere, $\delta(\Omega)=\delta(\theta) \delta(\phi) / \sqrt{g_{2}}=\delta(\cos \theta-1) \delta(\phi)$, which peaks at $\theta=0$. Substituting (13) and (15) in the first of (16) we recover just the background equation (5), linking the Hubble parameter $H$ and the brane tension $\lambda$. The new equation for the wave profile $f$ comes from the $u u$ component of (16). Indeed, direct substitution of (13) and (15) yields a linear field equation for the wave profile:

$$
\begin{array}{r}
\frac{M_{5}^{3}}{M_{4}^{2} H^{2}}\left(\partial_{z}^{2} f-3 \epsilon H \partial_{|z|} f+H^{2}\left(\Delta_{2} f+2 f\right)\right)+ \\
+\left(\Delta_{2} f+2 f\right) \delta(z)=\frac{2 p}{M_{4}^{2}} \delta(\Omega) \delta(z)
\end{array}
$$

The remaining components of the second of (16) again reduce to the background equation (5). Thus (17) fully determines the shock wave profile $f$. Note that in the limit $M_{5} \rightarrow 0$ the bulk derivatives disappear, and we can factor out $\delta(z)$ to recover the correct $4 \mathrm{D}$ de Sitter equation of [22, 24].

Now we can turn to solving (17). It turns out that one has to carefully implement the boundary conditions for the single particle problem (17) in order to avoid additional singularities that would arise because the 2 -sphere on which the particle propagates is compact 24]. These boundary conditions are handled in the simplest way by adding an extra particle with the same momentum $p$, running in the opposite direction in the static patch (7) [22, 23]. In fact, this two-source solution correctly represents the limit of infinite boost of the Schwarzschild-de Sitter geometry [22, 23]. So following [22, 23, 24] we add an extra term, $\frac{2 p}{M_{4}^{2}} \delta\left(\Omega^{\prime}\right) \delta(z)$, on the RHS of (17). Here $\delta\left(\Omega^{\prime}\right)$ is peaked on the opposite pole on the 2 -sphere, at $\theta=\pi$ (see Fig. 2). Then we can return to a single source by taking this solution and multiplying it by $\Theta(\pi / 2-\theta)$ as in 24].

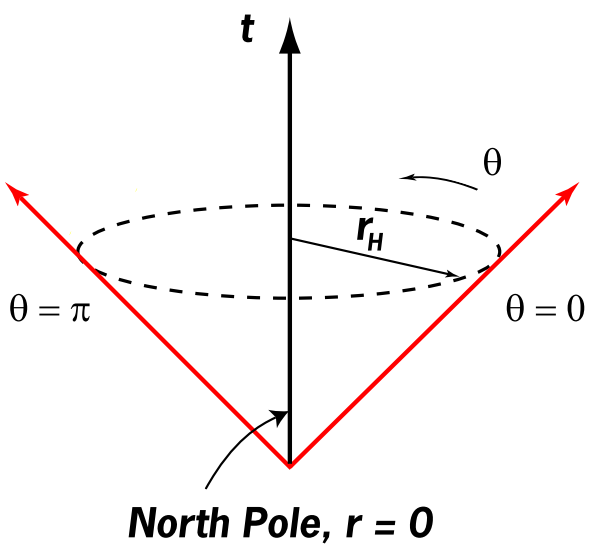

FIG. 2: Trajectories of two photons in the reference frame of an observer at rest on the North Pole, at $r=0$ in the static patch. The photons move along opposite directions on the $S^{2}$, at coordinate distance $r_{H}=1 / H$ from the pole. We are explicitly depicting the polar angle $\theta$ and suppressing the azimuthal angle $\phi$ on $S^{2}$. 
So let us consider eq. (17) with two photons, when one of them moves along $\theta=0$ and the other along $\theta=\pi$ :

$$
\begin{aligned}
& \frac{M_{5}^{3}}{M_{4}^{2} H^{2}}\left(\partial_{z}^{2} f-3 \epsilon H \partial_{|z|} f+H^{2}\left(\Delta_{2} f+2 f\right)\right)+ \\
& \quad+\left(\Delta_{2} f+2 f\right) \delta(z)=\frac{2 p}{M_{4}^{2}}\left(\delta(\Omega)+\delta\left(\Omega^{\prime}\right)\right) \delta(z) .
\end{aligned}
$$

Since the sources on the RHS are $\delta(\Omega)=\delta(\cos \theta-1) \delta(\phi)$ and $\delta\left(\Omega^{\prime}\right)=\delta(\cos \theta+1) \delta(\phi)$, they can be decomposed in terms of spherical harmonics as

$$
\begin{aligned}
\delta(\Omega) & =\sum_{l=0}^{\infty} \sum_{m=-l}^{l} Y_{l m}^{*}(0,0) Y_{l m}(\theta, \phi), \\
\delta\left(\Omega^{\prime}\right) & =\sum_{l=0}^{\infty} \sum_{m=-l}^{l} Y_{l m}^{*}(0,0) Y_{l m}(\pi-\theta, \phi) .
\end{aligned}
$$

Since we chose the photon trajectories to be along $\theta=0$ and $\theta=\pi$, we can use the addition theorem for spherical harmonics to replace them with Legendre polynomials $P_{l}(\cos \theta)$ in the expansion for the wave profile. Indeed, from $\sum_{m=-l}^{l} Y_{l m}^{*}(0,0) Y_{l m}(\theta, \phi)=\frac{2 l+1}{4 \pi} P_{l}(\cos \theta)$ and the similar equation for the $\theta \rightarrow \pi-\theta$ terms, and linearity of (18), we deduce that the solutions are of the form

$$
f=\sum_{l=0}^{\infty}\left(f_{l}^{(+)}(z) P_{l}(\cos \theta)+f_{l}^{(-)}(z) P_{l}(-\cos \theta)\right) .
$$

Here $f_{l}^{( \pm)}(z)$ are the bulk wave functions; $f_{l}^{(+)}$is sourced by the photon at $\theta=0$ and $f_{l}^{(-)}$by the photon at $\theta=\pi$. By orthogonality and completeness of Legendre polynomials, using the expansions for the $\delta$-functions from above, the field equation (18) yields the same differential equation for the modes $f_{l}^{( \pm)}(z)$ :

$$
\begin{aligned}
& \partial_{z}^{2} f_{l}-3 \epsilon H \partial_{|z|} f_{l}+H^{2}(2-l(l+1)) f_{l}= \\
& \quad=\frac{M_{4}^{2} H^{2}}{M_{5}^{3}}\left(\frac{(2 l+1) p}{2 \pi M_{4}^{2}}-(2-l(l+1)) f_{l}\right) \delta(z) .
\end{aligned}
$$

We should interpret $\delta$-function on the RHS by pillbox integration as a matching condition for the first derivatives of $f_{l}^{( \pm)}$on the brane, recalling also the orbifold symmetry which enforces $f_{l}(-z)=f_{l}(z)$. This yields the boundary value problem for $f_{l}$ on the brane:

$$
\begin{aligned}
& \partial_{z}^{2} f_{l}-3 \epsilon H \partial_{z} f_{l}+H^{2}(2-l(l+1)) f_{l}=0, \\
& f_{l}(-z)=f_{l}(z), \\
& f_{l}^{\prime}(0)+\frac{2-l(l+1)}{\mathrm{g}} H f_{l}(0)=\frac{H}{\mathrm{~g}} \frac{2 l+1}{2 \pi M_{4}^{2}} p .
\end{aligned}
$$

where $\mathrm{g}=2 M_{5}^{3} /\left(M_{4}^{2} H\right)=1 /\left(H r_{c}\right)$ (see [1] ). Since both $f_{l}^{( \pm)}$solve the same boundary value problem, $f_{l}^{(+)}=$ $f_{l}^{(-)}=f_{l}$. Because $P_{l}(-x)=(-1)^{l} P_{l}(x)$, the solution will be an expansion only in even-indexed polynomials
$P_{2 l}(\cos \theta)$, circumventing the unphysical $4 \mathrm{D}$ singularities in $l=1$ terms like in 22, 24]:

$$
f=2 \sum_{l=0}^{\infty} f_{2 l}(z) P_{2 l}(\cos \theta) .
$$

We still need to specify the boundary conditions for $f$ in the limit $z \rightarrow \infty$. We do so by requiring square integrability in the bulk, so that the solutions are localized on the brane. To see how to implement square integrability on the modes $f_{l}$, it is sufficient to consider the simpler example of a free massless bulk scalar field, with action

$$
S=-\int d^{5} x \sqrt{g_{5}}(\nabla \chi)^{2} .
$$

In terms of the conformal geometry (12), the action is

$$
S=-\int d z d^{4} x \sqrt{g_{4}} \Omega^{3}(\bar{\nabla} \chi)^{2},
$$

where $\Omega=\exp (-\epsilon H|z|)$. Therefore, the norm for the expansion of $\chi$ in terms of the $4 \mathrm{D}$ modes satisfies

$$
\left\|\chi_{\alpha}\right\|^{2} \propto \int d z e^{-3 \epsilon H|z|} \chi_{\alpha}^{2}
$$

We are interested in the modes of $\chi$ which are simple exponentials of $|z|$, because from (22) it follows that $f_{l}$ 's are such simple exponentials. Hence let $\chi_{\alpha}(x) \propto e^{-\alpha|z|}$; then from (26) we conclude that a mode will be normalizable only if $\alpha \geq(1-3 \epsilon H) / 2$. Solving the differential equation in (22) we see that the modes are of the form $\sim e^{[ \pm 2 l-(\mp 1-3 \epsilon) / 2] H|z|}$. Hence we should retain only the sub-leading bulk mode which is square-integrable, or equivalently, localized on the brane:

$$
f_{l}=A_{l} e^{-[2 l+(1-3 \epsilon) / 2] H|z|} .
$$

We can now write down explicitly the series solution of (22) which satisfies these boundary conditions. Substituting (27) in the last of (22) and solving the ensuing algebraic equation for $A_{l}$, we find

$$
\begin{aligned}
f(\Omega, z)=-\frac{p}{2 \pi M_{4}^{2}} & \sum_{l=0}^{\infty} \frac{4 l+1}{\left(2 l-1+\frac{(1-\epsilon) \mathrm{g}}{2}\right)\left(l+1+\frac{(1+\epsilon) \mathrm{g}}{4}\right)} \\
& \times e^{-[2 l+(1-3 \epsilon) / 2] H|z|} P_{2 l}(\cos \theta),(28)
\end{aligned}
$$

As a check, in the limit $\mathrm{g}=0$, at $z=0$ this reduces to the $4 \mathrm{D}$ series solution of 22 for both $\epsilon= \pm 1$. We can write the series (28) in the integral form. Defining $\tau=e^{-H|z|}$ and $x=\cos \theta$ and factorizing the coefficients of the expansion, we first find

$$
\begin{aligned}
f(\Omega, z) & =-\frac{[3-(1-\epsilon) \mathrm{g}] p}{(3+\epsilon \mathrm{g}) \pi M_{4}^{2}} \sum_{l=0}^{\infty} \frac{\tau^{2 l+(1-3 \epsilon) / 2}}{2 l-1+\frac{(1-\epsilon) \mathrm{g}}{2}} P_{2 l}(x) \\
& -\frac{[3+(1+\epsilon) \mathrm{g}] p}{2(3+\epsilon \mathrm{g}) \pi M_{4}^{2}} \sum_{l=0}^{\infty} \frac{\tau^{2 l+(1-3 \epsilon) / 2}}{l+1+\frac{(1+\epsilon) \mathrm{g}}{4}} P_{2 l}(x) .
\end{aligned}
$$


In the limit $\mathrm{g}=1$ the $l=0$ term in the first series diverges for $\epsilon=-1$. There are no divergences in the case $g=3$, as we see from eq. (28). We postpone the discussion of the limit $\mathrm{g}=1$ until the next section, and for the remainder of this section take $g \neq 1$.

Recalling the definition of the generating function for Legendre polynomials, $\left(1-2 x \tau+\tau^{2}\right)^{-1 / 2}=\sum_{l=0}^{\infty} P_{l}(x) \tau^{l}$, and using

$$
\lim _{\varepsilon \rightarrow 0} \int_{\varepsilon}^{\tau} d \vartheta \vartheta^{l+\beta}=\frac{\tau^{l+\beta+1}}{l+\beta+1}-\lim _{\varepsilon \rightarrow 0} \int^{\varepsilon} d \vartheta \vartheta^{l+\beta}
$$

to regulate divergences when $l+\beta \leq-1$, we integrate the generating function expansion over $\tau$. This yields the identity

$$
\begin{aligned}
& \sum_{l=0}^{\infty} \frac{\tau^{2 l+\beta+1}}{2 l+\beta+1} P_{2 l}(x)= \\
& \frac{1}{2} \lim _{\varepsilon \rightarrow 0}\left\{2 \sum_{l=0}^{l_{\max } \leq-(1+\beta) / 2} P_{2 l}(x) \int^{\varepsilon} d \vartheta \vartheta^{2 l+\beta}+\right. \\
& \left.+\int_{\varepsilon}^{\tau} d \vartheta \vartheta^{\beta}\left(\frac{1}{\sqrt{1-2 x \vartheta+\vartheta^{2}}}+\frac{1}{\sqrt{1+2 x \vartheta+\vartheta^{2}}}\right)\right\}
\end{aligned}
$$

Applying eq. (31) to eq. (29), after straightforward manipulations and performing explicitly the limit $\varepsilon \rightarrow 0$ we find

$$
\begin{aligned}
& f(\Omega, z)=\frac{p}{2 \pi M_{4}^{2}} \frac{\tau^{\frac{1-3 \epsilon}{2}}}{1-\frac{1-3 \epsilon}{4} \mathrm{~g}}-\frac{p}{2(3+\epsilon \mathrm{g}) \pi M_{4}^{2}} \times \\
& \times \int_{0}^{\tau} d \vartheta\left(\frac{1}{\sqrt{1-2 x \vartheta+\vartheta^{2}}}+\frac{1}{\sqrt{1+2 x \vartheta+\vartheta^{2}}}-2\right) \\
& \times\left(\frac{[3-(1-\epsilon) \mathrm{g}] \vartheta^{\frac{\mathrm{g}(1-\epsilon)-4}{2}}}{\tau^{\frac{(\mathrm{g}-3)(1-\epsilon)}{2}}}+\frac{[3+(1+\epsilon) \mathrm{g}] \vartheta^{\frac{\mathrm{g}(1+\epsilon)+2}{2}}}{\tau^{\frac{(\mathrm{g}+3)(1+\epsilon)}{2}}}\right) .
\end{aligned}
$$

When $\mathrm{g} \neq 1$ the integral (32) is finite and well-defined everywhere except at $x= \pm 1$, where it has the usual short-distance singularities, because it is a Green's function in the transverse spatial directions and $x= \pm 1$ is where the sources are located. We remove the spurious poles in the limit $\varepsilon \rightarrow 0$ in the integral representation of (32), arising from the implementation of the regulator (30), (31), by the introduction of the factor -2 in the second line of the integrand of (32).

For completeness' sake, we note that the integral (32) can be expressed in terms of the two-variable hypergeometric functions $F_{1}\left(\alpha, \beta_{1}, \beta_{2}, \gamma ; p, q\right)$ [37], which are defined by the integral representation

$$
\begin{aligned}
F_{1}\left(\alpha, \beta_{1},\right. & \left.\beta_{2}, \alpha+\mu ; p, q\right)=\frac{1}{B(\alpha, \mu)} \int_{0}^{1} d \zeta \times \\
& \times \zeta^{\alpha-1}(1-\zeta)^{\mu-1}(1-p \zeta)^{-\beta_{1}}(1-q \zeta)^{-\beta_{2}}
\end{aligned}
$$

valid when $\operatorname{Re} \alpha>0, \operatorname{Re} \mu>0$. As an illustration, we will only consider here the shock wave profile $f$ on the brane, at $z=0$ (i.e. $\tau=1$ ), on the self-inflating branch $\epsilon=-1$. Similar derivation can also be performed for the normal branch $\epsilon=1$. We will split up the integral in (32) into a sum of two integrals, with terms $\propto\left(1 \mp 2 x \vartheta+\vartheta^{2}\right)^{-1 / 2}-1$, and evaluate each integral separately by using the Euler substitutions $1+\sqrt{2(1 \mp x)} \zeta=\sqrt{1 \mp 2 x \vartheta+\vartheta^{2}}+\vartheta$. To see how the hypergeometric functions in (33) emerge, consider e.g.

$$
I=\int_{0}^{1} d \vartheta\left(\frac{1}{\sqrt{1-2 x \vartheta+\vartheta^{2}}}-1\right)\left(\frac{3-2 \mathrm{~g}}{3-\mathrm{g}} \vartheta^{\mathrm{g}-2}+\frac{3}{3-\mathrm{g}} \vartheta\right) \text {, }
$$

and plug in $1+\sqrt{2(1-x)} \zeta=\sqrt{1-2 x \vartheta+\vartheta^{2}}+\vartheta$. After straightforward algebra, we can rewrite the integral (34), after introducing $Q=-\sqrt{(1-x) / 2}=-\sin (\theta / 2)$, as

$$
\begin{aligned}
I=\int_{0}^{1} d \zeta \zeta \frac{x+Q \zeta}{(\zeta-Q)^{2}} & \left\{\frac{3-2 \mathrm{~g}}{3-\mathrm{g}}\left(\frac{\zeta(1-Q \zeta)}{\zeta-Q}\right)^{\mathrm{g}-2}\right. \\
& \left.+\frac{3}{3-\mathrm{g}} \frac{\zeta(1-Q \zeta)}{\zeta-Q}\right\} .
\end{aligned}
$$

Breaking up the integrand into the powers of the kind that enter in (33) we can rewrite (35) as

$$
\begin{aligned}
I & =\frac{(3-2 \mathrm{~g}) \Gamma(\mathrm{g}-2)}{(3-\mathrm{g}) \Gamma(\mathrm{g}-1)} \frac{x F_{1}\left(\mathrm{~g}-2,2-\mathrm{g}, \mathrm{g}, \mathrm{g}-1 ; Q, \frac{1}{Q}\right)}{(-Q)^{\mathrm{g}}} \\
& -\frac{(3-2 \mathrm{~g}) \Gamma(\mathrm{g}-1)}{(3-\mathrm{g}) \Gamma(\mathrm{g})} \frac{F_{1}\left(\mathrm{~g}-1,2-\mathrm{g}, \mathrm{g}, \mathrm{g} ; Q, \frac{1}{Q}\right)}{(-Q)^{\mathrm{g}-1}} \\
& +\frac{3}{(3-\mathrm{g})} \frac{x F_{1}\left(1,-1,3,2 ; Q, \frac{1}{Q}\right)}{(-Q)^{3}} \\
& -\frac{3}{2(3-\mathrm{g})} \frac{F_{1}\left(2,-1,3,3 ; Q, \frac{1}{Q}\right)}{(-Q)^{2}} .
\end{aligned}
$$

The remaining integrals in (32) can be found from (36) by substituting $x \rightarrow-x$. Then, using $P=-\sqrt{(1+x) / 2}=$ $-\cos (\theta / 2)$, we finally obtain

$$
\begin{aligned}
& f(\Omega)=\frac{p}{2 \pi M_{4}^{2}} \frac{1}{1-\mathrm{g}}-\frac{p}{2 \pi(3-\mathrm{g}) M_{4}^{2}}\{ \\
& \frac{(3-2 \mathrm{~g}) \Gamma(\mathrm{g}-2)}{\Gamma(\mathrm{g}-1)} \frac{x F_{1}\left(\mathrm{~g}-2,2-\mathrm{g}, \mathrm{g}, \mathrm{g}-1 ; Q, \frac{1}{Q}\right)}{(-Q)^{\mathrm{g}}} \\
- & \frac{(3-2 \mathrm{~g}) \Gamma(\mathrm{g}-1)}{\Gamma(\mathrm{g})} \frac{F_{1}\left(\mathrm{~g}-1,2-\mathrm{g}, \mathrm{g}, \mathrm{g} ; Q, \frac{1}{Q}\right)}{(-Q)^{\mathrm{g}-1}} \\
+ & 3\left(\frac{x F_{1}\left(1,-1,3,2 ; Q, \frac{1}{Q}\right)}{(-Q)^{3}}-\frac{F_{1}\left(2,-1,3,3 ; Q, \frac{1}{Q}\right)}{2(-Q)^{2}}\right) \\
- & \frac{(3-2 \mathrm{~g}) \Gamma(\mathrm{g}-2)}{\Gamma(\mathrm{g}-1)} \frac{x F_{1}\left(\mathrm{~g}-2,2-\mathrm{g}, \mathrm{g}, \mathrm{g}-1 ; P, \frac{1}{P}\right)}{(-P)^{\mathrm{g}}} \\
- & \frac{(3-2 \mathrm{~g}) \Gamma(\mathrm{g}-1)}{\Gamma(\mathrm{g})} \frac{F_{1}\left(\mathrm{~g}-1,2-\mathrm{g}, \mathrm{g}, \mathrm{g} ; P, \frac{1}{P}\right)}{(-P)^{\mathrm{g}-1}} \\
- & \left.3\left(\frac{x F_{1}\left(1,-1,3,2 ; P, \frac{1}{P}\right)}{(-P)^{3}}+\frac{F_{1}\left(2,-1,3,3 ; P, \frac{1}{P}\right)}{2(-P)^{2}}\right)\right\} .
\end{aligned}
$$

In the expressions for the hypergeometric functions one would encounter an additional, spurious, logarithmic singularity. This singularity is manifest in the integrals (34) 
or (35) in the lower limit of integration. However, after Euler substitutions for integration variables, one has to carefully relate the singularities in (34) and (35), recalling that these integrals are defined as principal values of integration over $\vartheta$, in accordance with the regularization prescription in (30). After this is done, these spurious logarithmic singularities cancel precisely between the $Q$ and $P$ dependent terms in (37), leaving only the physical short distance singularities carried by the null sources on the brane. We will revisit this more closely in section V.

\section{RESONANCE ON THE SELF-INFLATING BRANCH}

As we have mentioned above, the solution (29) for the shock wave displays a spectacular behavior on the selfinflating branch, $\epsilon=-1$, as $\mathrm{g} \rightarrow 1$. In this limit the tension vanishes, as can be seen from eq. (5). The $l=0$ term of the first sum has a pole there. No such poles arise on the self-inflating branch, where the series (29) is finite. What then does this divergence mean? To gain some insight we revisit the boundary value problem (22) for the $l=0$ mode but slightly relax the boundary conditions in the bulk, by retaining the $l=0$ solution which is not square-integrable as well. Writing this mode as

$$
f_{0}=A_{0} e^{-2 H|z|}+B_{0} e^{-H|z|},
$$

and solving for $A_{0}$ and $B_{0}$ we find that when $\mathrm{g}=1$ the mode $\propto A_{0}$ is an eigenmode of the homogeneous $(p=0)$ boundary value problem (22), completely cancelling on the LHS of the last of eq. (22). This is the origin of the divergence in (29) for $g=1$ and mathematically is an example of the Fredholm's alternative: either the inhomogeneous differential equation or the associated homogeneous differential equation (i.e. with non-vanishing source or with vanishing source, respectively) have a solution, but not both at the same time. On the other hand, the mode $\propto B_{0}$ remains. Hence we could solve the last of (22) for it:

$$
B_{0}=\frac{p}{2 \pi M_{4}^{2}} .
$$

Because we have excluded the non-integrable bulk modes with $l>0$, this mode is the fastest-growing one in the limit $z \rightarrow \infty$ among all the allowed solutions of (22) for any $l$. Thus far from the brane the resulting wave profile behaves as

$$
f \rightarrow \frac{p}{\pi M_{4}^{2}} e^{-H|z|} .
$$

Hence as $z$ approaches the Cauchy horizon at $z \rightarrow \infty$ (see Fig. 10) the asymptotic metric (9) is

$$
\begin{aligned}
d s_{5}^{2} \rightarrow e^{-2 \epsilon H|z|}\{ & \frac{4 d u d v}{\left(1+H^{2} u v\right)^{2}}+\left(\frac{1-H^{2} u v}{1+H^{2} u v}\right)^{2} \frac{d \Omega_{2}}{H^{2}} \\
& \left.+d z^{2}\right\}-\frac{4 p}{\pi M_{4}^{2}} e^{-\epsilon H|z|} \delta(u) d u^{2} .
\end{aligned}
$$

Because the last term remains finite, the shock wave is not localized to the brane, but extends deep into the bulk, as anticipated. This solution carries a lot of energy and curvature into the bulk, similarly to the gravitational pp-waves in the Randall-Sundrum setup, studied in [38]. Indeed, we can rewrite the asymptotic metric (41) expressed in coordinates $u, v, z$ in terms of the original static patch coordinates $r, t, w$ (see the text above eq. (7)), and transform further to the spherical polar coordinates in the bulk far from the brane, defined by

$$
\begin{aligned}
w & =\frac{1}{H}-\sqrt{\rho^{2}-\tau^{2}} \\
r & =\frac{1}{H} \frac{\rho}{\sqrt{\rho^{2}-\tau^{2}}} \sin \varphi \\
\sinh (H t) & =\frac{\tau}{\sqrt{\rho^{2} \cos ^{2} \varphi-\tau^{2}}} .
\end{aligned}
$$

Then the asymptotic metric (41) becomes

$d s_{5}^{2} \rightarrow-d \tau^{2}+d \rho^{2}+\rho^{2} d \Omega_{3}-\frac{8 p \sqrt{\rho^{2}-\tau^{2}}}{\pi M_{4}^{2} H} \delta\left(\mathcal{X}^{2}-1\right) d \mathcal{X}^{2}$.

where $\mathcal{X}=\frac{\rho \cos \varphi+\tau}{\sqrt{\rho^{2}-\tau^{2}}+\rho \sin \varphi}$, and $d \Omega_{3}=d \varphi^{2}+\sin ^{2} \varphi d \Omega_{2}$ is the line element on a unit 3-sphere. Since this asymptotic metric is invariant under $S^{2}$ rotations, the solution looks like an imploding shell of energy in Minkowski space. For a hint of how such a shell is generated, move away from $\mathrm{g}=1$ and reconsider the boundary value problem (22) while still keeping the non-integrable bulk wave function in the $l=0$ mode, as in eq. (38). Then (22) relates $A_{0}$ and $B_{0}$ according to

$$
B_{0}=\frac{1}{2-\mathrm{g}}\left(\frac{p}{2 \pi M_{4}^{2}}-2(1-\mathrm{g}) A_{0}\right) .
$$

Thus we see that when $\mathrm{g} \neq 1$, the localized mode $\propto A_{0}$ can absorb fully the effect of the source $\propto p$. This is energetically favorable, because the non-integrable mode $\propto B_{0}$ decouples because it has vanishing overlap with the brane where the source is located. Hence we can set $B_{0}=0$ and forget about it. However in the limit $\mathrm{g}=1$ the modifications of gravity occur at the scale equal to the cosmological horizon on the brane. Brane and bulk begin to "resonate": in a time-dependent problem, as $\mathrm{g} \rightarrow 1$ slowly, the localized $l=0$ mode would grow ever larger, spreading further out into the bulk. It would develop a larger overlap with the bulk graviton, so that it can produce delocalized gravitons in order to shed the energy which drives its unbounded growth. In this way as $\mathrm{g} \rightarrow 1$ energy could escape into the bulk. This indicates an onset of a dramatic new instability, which warrants further investigation.

\section{SHOCK WAVES AT SHORT DISTANCES}

In this section we consider $f$ on the brane, when $z=0$ (i.e. $\tau=1$ ), at transverse distances $\mathcal{R}$ well inside the cosmological horizon, $\mathcal{R} \ll H^{-1}$. Note that we can rewrite 
eq. (5) as $(1+\epsilon \mathrm{g}) H^{2}=\lambda /\left(3 M_{4}^{2}\right)$. This shows that on the normal branch $\mathrm{g}$ can vary between zero, in which case we recover the $4 \mathrm{D}$ limit, and infinity, when we recover the 5D limit, with the "resonance" discussed in detail in the previous section at $\mathrm{g}=1$. On the other hand, on the selfinflating branch, $\mathrm{g} \leq 1$ or $r_{c} \geq 1 / H$, as long as $\lambda \geq 0$. In the limit $\mathrm{g} \rightarrow 1$ on the self-inflating brane, as mentioned before, the tension vanishes.

Let us start with $\mathrm{g}=0$. In this case (32) is identical to the $4 \mathrm{D}$ case on both branches. Indeed, it can be expressed as $f=\frac{p}{2 \pi M_{4}^{2}}\left(1-I_{-}-I_{+}\right)$where $I_{\mp}$ are the integrals

$$
I_{\mp}=\int_{0}^{1} d \vartheta\left(\frac{1}{\vartheta^{2}}+\vartheta\right)\left(\frac{1}{\sqrt{1 \mp 2 x \vartheta+\vartheta^{2}}}-1\right),
$$

which obey $I_{+}(x)=I_{-}(-x)$. Hence as in section III it is enough to evaluate one, say $I_{-}$. Using the Euler substitution $\xi=\sqrt{1-2 x \vartheta+\vartheta^{2}}+\vartheta$ we can rewrite the integral $I_{-}$as

$$
\begin{aligned}
I_{-} & =4 x \int_{1+\varepsilon_{-}}^{1+\sqrt{2(1-x)}} \frac{d \xi}{(\xi+1)^{2}(\xi-1)} \\
& -2 \int_{1}^{1+\sqrt{2(1-x)}} \frac{d \xi}{(\xi+1)^{2}} \\
& -\frac{1}{4} \int_{1}^{1+\sqrt{2(1-x)}} d \xi \frac{(z-1)^{2}(z+1)}{(\xi-x)^{2}} \\
& +\frac{1+x}{4} \int_{1}^{1+\sqrt{2(1-x)}} d \xi \frac{(z-1)^{2}(z+1)}{(\xi-x)^{3}}
\end{aligned}
$$

where in the first line we have replaced $1 \rightarrow 1+\varepsilon_{-}$in the lower limit of integration in order to regulate a spurious logarithmic singularity, which we have discussed in section III. A direct evaluation of the integrals yields

$$
I_{-}=x \ln \frac{2}{\varepsilon_{-}}-x-\frac{1}{2},
$$

and thus, using $I_{+}(x)=I_{-}(-x)$, and noting that the regulator is now $\varepsilon_{+}$, also

$$
I_{+}=-x \ln \frac{2}{\varepsilon_{+}}+x-\frac{1}{2},
$$

such that the final result for $I=I_{+}+I_{-}$is

$$
I=x \ln \frac{\varepsilon_{+}}{\varepsilon_{-}}-1 .
$$

To determine the ratio of the regulators $\frac{\varepsilon_{+}}{\varepsilon_{-}}$, recall that they are the image of the regulator $\varepsilon$ for the variable $\vartheta$ under the Euler substitution for the integration variables in $I_{ \pm}$. That yields $\varepsilon_{\mp}=(1 \mp x) \varepsilon$, so that

$$
\frac{\varepsilon_{+}}{\varepsilon_{-}}=\frac{1+x}{1-x} .
$$

Therefore the singularities indeed cancel out, and

$$
I=x \ln \left[\frac{1+x}{1-x}\right]-1 .
$$

Substituting this in the equation for the shock wave profile finally yields

$$
f_{4 D}(\Omega)=\frac{p}{2 \pi M_{4}^{2}}\left(2-x \ln \left[\frac{1+x}{1-x}\right]\right) .
$$

The metric transverse to the null particle on the brane is $\left.d s_{2}^{2}\right|_{z=u=0}=d \Omega_{2} / H^{2}$. Therefore the proper "radial" transverse distance for small polar angles $\theta$ is $\mathcal{R} \simeq \theta / H$, so that $x=1-H^{2} \mathcal{R}^{2} / 2$. Thus the shock wave profile (52) reduces precisely to the flat $4 \mathrm{D}$ solution [17, 18]: up to $\mathcal{O}\left(\mathcal{R}^{2} / H^{-2}\right)$ corrections, using the sign conventions of 22, 24], we find

$$
f_{4 D}(\Omega)=\frac{p}{\pi M_{4}^{2}}+\frac{p}{\pi M_{4}^{2}} \ln \left(\frac{\mathcal{R}}{2 H^{-1}}\right) .
$$

As long as it is finite, the numerical value of the constant term in this equation is physically unimportant since a finite constant term in the shock wave profile in a flat $4 \mathrm{D}$ spacetime can always be changed by a simple $4 \mathrm{D}$ diffeomorphism [18]. We cannot write down the integrals which incorporate the $\mathcal{O}(\mathrm{g})$ corrections in closed form because they contain terms like $\int d \zeta \ln (1+b \zeta) / \zeta$ [37]. Nevertheless, it is easy to extract their short-distance effects: at short distances the singular $\mathcal{O}(\mathrm{g})$ powers in the integrand precisely cancel out. In the remainder, since the leading order singularity in (32) is logarithmic around $\mathrm{g}=0$, the additional logarithms soften the corrections further, and render them finite as $\mathcal{R} \rightarrow 0$. Hence at short distances the solution looks exactly the same as in $4 \mathrm{D}$, and the corrections are negligible when $\mathcal{R}<H^{-1} / \mathrm{g}$.

In fact this behavior persists for any finite value of $g$. We can see this from identifying the leading contribution in (32) at short distances. Rewriting the integrals (32) by using the same substitutions $\xi=\sqrt{1 \mp 2 x \vartheta+\vartheta^{2}}+\vartheta$ and looking at the limit $1-x \ll 1$, we identify the leading singularity. After cancelling the spurious terms as above, for all values of $g \neq 1$ and $\epsilon$ the leading singularity at short distances comes from the term

$$
-\frac{p}{\pi M_{4}^{2}} \int_{1}^{1+\sqrt{2(1-x)}} \frac{d \xi}{\xi-x}=\frac{p}{\pi M_{4}^{2}} \ln \sqrt{\frac{1-x}{2}}+\text { finite . }
$$

Substituting $x=1-H^{2} \mathcal{R}^{2} / 2$, we recover the leading logarithm in (53). The corrections to this result at larger distances in general differ from the corrections which arise when one expands the $4 \mathrm{D}$ de Sitter solution (52) around the short distance limit describing shock waves in asymptotically flat space (53), where one finds corrections to come as even powers of $H \mathcal{R}$. In DGP, however, the corrections in (55) will arise as odd powers of $H \mathcal{R}$ as well, starting with the linear term. This signals the presence of the fifth dimension concealed by the "gravity filter". Therefore in general we will have

$$
\begin{aligned}
f(\Omega)= & \frac{p}{\pi M_{4}^{2}}\left(\left(1+a_{1} H^{2} \mathcal{R}^{2}+\ldots\right) \ln \left(\frac{\mathcal{R}}{2 H^{-1}}\right)\right. \\
& \left.+ \text { const }+b_{1} H \mathcal{R}+b_{2} H^{2} \mathcal{R}^{2}+\ldots\right),
\end{aligned}
$$


where the coefficients $a_{k}, b_{k}$ are numbers that can be computed explicitly for given values of $g$ and $\epsilon$, and we have seen that e.g. $b_{1} \sim \mathrm{g}$.

To see how $5 \mathrm{D}$ gravity reemerges, we can take the limit $g \rightarrow \infty$ on the normal branch $\epsilon=1$, keeping $M_{5}$ fixed. This removes the brane-localized terms $\propto M_{4}$ from the action and neutralizes the "gravity filter". In this case the background solution reduces to the inflating brane in 5D Minkowski bulk 28]. From eq. (29) we see that the shock wave profile on the brane at $z=0$ in the limit $g \rightarrow \infty$ reduces to

$$
f_{5 D}(\Omega)=-\frac{p H}{\pi M_{5}^{3}} \sum_{l=0}^{\infty} P_{2 l}(x)-\frac{3 p H}{2 \pi M_{5}^{3}} \sum_{l=0}^{\infty} \frac{P_{2 l}(x)}{2 l-1},
$$

where we have used $M_{4}^{2} \mathrm{~g}=2 M_{5}^{3} / H$. We can evaluate the first sum using the identity

$$
\sum_{l=0}^{\infty} P_{2 l}(x)=\frac{1}{\sqrt{8(1-x)}}+\frac{1}{\sqrt{8(1+x)}},
$$

and reduce the second sum to the integral representation

$$
\begin{aligned}
\sum_{l=0}^{\infty} \frac{P_{2 l}(x)}{2 l-1}=\frac{1}{2} & \int_{0}^{1} \frac{d \vartheta}{\vartheta^{2}}\left(\frac{\vartheta}{\sqrt{1-2 x \vartheta+\vartheta^{2}}}\right. \\
& \left.+\frac{\vartheta}{\sqrt{1+2 x \vartheta+\vartheta^{2}}}-2\right)-1 .
\end{aligned}
$$

Evaluating the integrals in much the same way as above, and substituting back in (56) we find

$$
\begin{aligned}
& f_{5 D}(\Omega)=-\frac{p H}{2 \sqrt{2} \pi M_{5}^{3}}\left(\frac{1}{\sqrt{1-x}}+\frac{1}{\sqrt{1+x}}\right) \\
& +\frac{3 p H}{4 \pi M_{5}^{3}} x \ln \left[\frac{\sqrt{\frac{1-x}{2}}\left(1+\sqrt{\frac{1-x}{2}}\right)}{\sqrt{\frac{1+x}{2}}\left(1+\sqrt{\frac{1+x}{2}}\right.}\right] \\
& +\frac{3 p H}{2 \pi M_{5}^{3}}\left(\sqrt{\frac{1-x}{2}}+\sqrt{\frac{1+x}{2}}\right) \text {. }
\end{aligned}
$$

At short transverse distances $x=1-H^{2} \mathcal{R}^{2} / 2$ ignoring a constant and higher powers of $\mathcal{R}$, we find the leading order behavior of $f_{5 D}$ to be

$$
f_{5 D}(\Omega)=-\frac{p}{2 \pi M_{5}^{3} \mathcal{R}}+\frac{3 p H}{4 \pi M_{5}^{3}} \ln \left(\frac{\mathcal{R}}{2 H^{-1}}\right) .
$$

The first term is precisely the $5 D$ shock wave solution considered in 19, 20]. The second term arises from the residual $4 \mathrm{D}$ graviton zero mode which persists on the normal branch in the limit $\mathrm{g} \rightarrow \infty$ because the bulk volume remains finite. Its perturbative coupling is set by the effective $4 \mathrm{D}$ Planck scale $M_{4 \text { eff }}^{2}$, which can be computed from the normalization of the zero mode graviton wave function in the bulk [36, 39] as

$$
M_{4 \text { eff }}^{2}=2 M_{5}^{3} \int_{0}^{\infty} d z \Omega^{3 / 2}=\frac{4 M_{5}^{3}}{3 H} .
$$

Substituting this in the formula for the $4 \mathrm{D}$ shock wave profile at short distances (53) exactly reproduces the second term in (60). Notice the relative sign difference between the two terms on the RHS of (60). This is necessary in order for both to yield an attractive force $\propto-\vec{\nabla} f$ on a test particle in the shock wave background. Therefore the result (60) precisely meets the expectations for the shock wave profile arising in a theory with both a $5 \mathrm{D}$ and $4 \mathrm{D}$ graviton modes in flat bulk. Because the two gravitons are equally coupled, the theory looks effectively five-dimensional at all sub-horizon distances, where the inverse power of $\mathcal{R}$ in (60) wins over the logarithm. Hence indeed in this limit the effects of the 4D graviton are completely negligible inside the cosmological horizon, and the "gravity filter" has been turned off.

This analysis clearly demonstrates how the "gravity filter" of [1] works even beyond perturbation theory. It is encoded in the behavior of the coefficients in (29), which decrease with the index $l$ of the Legendre polynomials. Because the graviton momentum on a transverse 2-sphere along de Sitter brane is proportional to the index of the polynomial, $q \sim H l$, the modes with $q>\mathrm{g} H \simeq 1 / r_{c}$ have amplitudes suppressed by $q$. Hence the short-distance divergences of the series can be no worse than a logarithm for any finite value of $\mathrm{g}$. Indeed, at short distances $\mathcal{R} \leq r_{c} \simeq H^{-1} / \mathrm{g}$, our shock waves are exactly the same as in 4D GR. For very low momenta the amplitudes are not suppressed, and so the shock wave profile changes towards $5 \mathrm{D}$ behavior at large distances $\mathcal{R} \geq H^{-1} / \mathrm{g}$. The limit $\mathrm{g} \rightarrow \infty$ on the normal branch illustrates this explicitly: when $r_{c} \rightarrow 0$ all modes with finite momenta remain unsuppressed and we recover $5 \mathrm{D}$ gravity.

\section{PLANCKIAN SCATTERING}

In this section we explore the scattering of two relativistic particles on the brane, $m_{k} / p_{k} \ll 1$, whose energymomenta $p_{1}, p_{2}$ reach beyond the Planck scale. In this regime, gravity will generically dominate over other interactions. Indeed in $4 \mathrm{D}$ the effective coupling of gravitons to the packets of stress-energy is $\alpha \simeq G_{N} s$, where $s=\left(p_{1}-p_{2}\right)^{2}=4 E^{2}$ is the Mandelstam $s$-parameter and $E$ the center-of-mass (COM) energy 40, 41, 42. It has been shown that even if it exceeds the Planck scale, the scattering cross section can still be reliably calculated using the methods of quantum field theory and GR 40, 41, 42, 43, 44]. Classical gravitational scattering has been studied before by D'Eath and Payne 45|, and the eikonal approximation calculations of [40, 41, 42] are consistent with these results when the impact parameter $b$ is larger than the center-of-mass Schwarzschild radius $\sim G_{N} \sqrt{s}$. The problem of Planckian scattering and black hole formation has been revived recently in the context of braneworld constructions, since if the fundamental scale of gravity is really low, it may be possible to form black holes in high energy processes in colliders [34, 35, 46, 47, 48, 49, 50]. Thus one might wonder if 
such processes would be different in interesting ways in the framework of brane-induced gravity.

However, in light of our findings above, it is clear that in the case of DGP with finite g, when the impact parameter $b$ is smaller than the scale of modification of gravity, $b<H^{-1} / \mathrm{g}$, Planckian gravitational scattering will be very well approximated by the conventional 4D GR description. The differences are suppressed by the powers of $m / p$ and $H \mathcal{R}$, which are both very small for highly energetic particles at short distances.

Let us now explicitly verify this. In the limit when the impact parameter is smaller than the DGP scale of modification of gravity, $b<H^{-1} / \mathrm{g}$, the scattering is mediated predominantly by the $5 \mathrm{D}$ modes with transverse momenta $q>1 / b>\mathrm{g} H$. These individual 5D modes couple to a brane-localized source with the effective coupling

$$
G_{N \text { eff }}(q) \sim \frac{1}{M_{4}^{2}} \frac{H}{q},
$$

as can be seen directly from (29), and is consistent with [1]. The latter term is the "gravity filter", which cuts off the influence of the modes with high transverse momentum. This is in contrast to what happens in the RandallSundrum models [39], where the modes carrying greater momentum compete more efficiently with the zero-mode graviton [35]. The couplings can be rewritten as

$$
G_{N e f f}(q) \sim \frac{1}{2 M_{5}^{3}} \frac{1}{\mathrm{~g} r_{c}} \frac{1}{r_{c} q}
$$

where the first factor is the usual $5 D$ bulk coupling, with the factor of 2 accounting for the orbifolding, the second factor is the "volume dilution" $\sim 1 /\left(\mathrm{g} r_{c}\right)=H$, and the last factor is the "filter". Hence in order for a mode mediating a process on the brane with a transverse momentum $q$ to be strongly coupled, the process should unravel at COM energies $E$ where $G_{N \text { eff }}(q) E^{2} \geq 1$, or therefore when $E \geq \sqrt{\frac{q}{H}} M_{4}$. This should be compared with the requirements for the processes in the bulk, where the filter does not operate, because they are mediated by delocalized modes, which become strongly coupled at much lower scales $E \geq M_{5}$.

Therefore we see that for the brane processes at energies $M_{4} / \sqrt{b H} \geq E \geq M_{4}$, all the individual modes with momenta $q>1 / b$ are in fact weakly coupled, with $G_{N \text { eff }}(q)<1$. However, the resonance mimicking the $4 \mathrm{D}$ graviton is strongly coupled. Indeed, this resonance couples to the brane-localized sources with the effective strength

$$
G_{N} \sim \sum_{q \geq 1 / b} G_{N e f f}(q) \sim \frac{1}{M_{4}^{2}} \int_{1 / b}^{\Lambda} \frac{d q}{q}=\frac{1}{M_{4}^{2}} \ln (\Lambda b)
$$

where the UV cutoff $\Lambda$ is the highest transverse momentum for which the particle scattering is meaningful, which we roughly estimate as $\Lambda \sim M_{4}$. Thus $G_{N} \sim \frac{1}{M_{4}^{2}}$ up to logarithmic corrections, and so at COM energies $E>M_{4}$ indeed $G_{N} E^{2}>1$, implying that the resonancemediated scattering is in the strong coupling regime, where it should dominate over other processes much like the conventional 4D gravity. This description should be reasonable as long as the impact parameter $b$ is greater than the Schwarzschild radius $\sim G_{N} E \simeq G_{N} \sqrt{s} 13$, where black holes are expected to form [40, 42, 45, 50], and where one would need to go beyond the eikonal approximation. It is interesting however that in the regime of COM energies $M_{4} \leq E \leq M_{4} / \sqrt{b H}$, and impact parameters $b \geq G_{N} E$, which yield the COM energy interval $M_{4} \leq E \leq M_{4}\left(M_{4} / H\right)^{1 / 3}$, the strongly coupled $4 \mathrm{D}$ graviton resonance is resolved into the individual weakly coupled bulk modes.

In this limit, the scattering amplitude can be extracted directly from the shock wave solutions [40]. For a shock wave profile $f(\Omega)$ on the brane at $z=0$, the formula relating the scattering amplitude in the eikonal approximation $\mathcal{A}(s, b)$, expressed via the eikonal $\delta_{E}(s, b)=$ $\ln \left(\frac{\mathcal{A}(s, b)}{2 i}\right)$, and the shock wave metric (9) is 35, 40]

$$
g_{u u}=\frac{p}{\pi} \delta(u) \frac{\delta_{E}(s, b)}{s} .
$$

Solving for $\delta_{E}(s, b)$ and substituting in the shock wave profile formula from (9) and (55), we obtain, taking into account our normalizations,

$$
\begin{array}{r}
\delta_{E}(s, b)=-\frac{s}{M_{4}^{2}}\left(\left(1+a_{1} H^{2} b^{2}+\ldots\right) \ln \left(\frac{b}{2 H^{-1}}\right)\right. \\
\left.+ \text { const }+b_{1} H b+b_{2} H^{2} b^{2}+\ldots\right),
\end{array}
$$

where $a_{k}, b_{k}$ are the same numerical coefficients as in eq. (55) (and $b_{1} \sim \mathrm{g}$ ). Thus clearly as long as $b \ll r_{c}=$ $H^{-1} / \mathrm{g}$, the deviations away from the expression in $4 \mathrm{D}$ GR are completely negligible. Hence as we have claimed above, the scattering will be very well approximated by the analysis in the conventional 4D theory [40, 41, 42]. As a consequence one expects also that the processes of black hole formation in high energy collisions would behave as in 4D GR [45, 46, 50], controlled by the low energy value of the Planck scale, $M_{4} \sim 10^{19} \mathrm{GeV}$, which is much too high to see such processes in forthcoming experiments at the LHC. This provides further illustration of the efficiency of the "gravity filter" [1].

\section{CONCLUSIONS}

In this paper we have given a detailed derivation and analysis of the exact gravitational shock wave metric in DGP models 1], first presented in our earlier paper [15]. The shock wave solutions provide an explicit and concrete example of the "gravity filter" mechanism of [1] beyond perturbation theory. Even on these exact solutions, the effects of the whole continuum of bulk graviton modes is dominated by a long range resonance, which mimics 
the standard 4D graviton out to distances $r_{c}=H^{-1} / \mathrm{g}$. This is because the bulk modes which can transfer large transverse momentum on the brane $q>\mathrm{g} H \simeq 1 / r_{c}$ have amplitudes suppressed by $q$. Hence at short distances $\mathcal{R} \leq r_{c}$ their influence is cut off, and the rate of divergence of the shock wave metrics can be no worse than a logarithm for any finite value of $g$, so that to the leading order the gravitational shock waves are exactly the same as in 4D GR. For very low momenta the amplitudes are not suppressed, leading to IR corrections which change the shock wave towards the $5 \mathrm{D}$ form at large distances $\mathcal{R} \geq H^{-1} / \mathrm{g}$. We have also demonstrated that the leading-order Planckian scattering behaves in exactly the same way as in 4D GR. The differences come with the powers of $m / p$ and $H \mathcal{R}$, and are very small for highly energetic particles at short distances. Further, we have noted a spectacular new channel for energy loss into the bulk on the self-inflating branch, which arises when gravity is modified at exactly the brane de Sitter radius.

We have argued earlier [15] that our exact shock waves may also provide for a new arena to explore the scalar graviton sector in DGP. In the relativistic limit pursued here and in [15] the scalar graviton has decoupled, in spite of the strong coupling issues that were encountered in nä̈ve perturbation theory [7, 9, 11, 12]. However one can imagine taking a very fast, but not ultra-relativistic observer who explores the field of a non-vanishing source mass. In this limit, one may treat the rest mass of the source as a perturbation of the shock wave geometry, and study how it sources the scalar graviton, and what happens to the graviton perturbation theory around the shock wave. This could reveal new aspects of modified gravitational physics in the IR, and help illuminate the status of effective 4D theory in DGP.

\section{Acknowledgements}

We thank S. Dimopoulos, G. Dvali, R. Emparan, G. Gabadadze, M. Luty, K. Sfetsos and L. Sorbo for useful discussions, and the Aspen Center for Physics for hospitality during the initiation of this work. NK was supported in part by the DOE Grant DE-FG03-91ER40674, in part by the NSF Grant PHY-0332258 and in part by a Research Innovation Award from the Research Corporation.
[1] G. R. Dvali, G. Gabadadze and M. Porrati, Phys. Lett. B 485 (2000) 208; G. R. Dvali and G. Gabadadze, Phys. Rev. D 63 (2001) 065007.

[2] R. Gregory, V. A. Rubakov and S. M. Sibiryakov, Phys. Rev. Lett. 84 (2000) 5928.

[3] G. R. Dvali, G. Gabadadze and M. Porrati, Phys. Lett. B 484 (2000) 129; C. Csaki, J. Erlich, T. J. Hollowood and J. Terning, Phys. Rev. D 63 (2001) 065019; L. Pilo, R. Rattazzi and A. Zaffaroni, JHEP 0007 (2000) 056.

[4] N. Arkani-Hamed, H. C. Cheng, M. A. Luty and S. Mukohyama, JHEP 0405 (2004) 074; N. Arkani-Hamed, H. C. Cheng, M. Luty and J. Thaler, hep-ph/0407034

[5] H. van Dam and M. J. G. Veltman, Nucl. Phys. B 22 (1970) 397; V. I. Zakharov, JETP Lett. 12 (1970) 312.

[6] A. I. Vainshtein, Phys. Lett. B 39 (1972) 393.

[7] C. Deffayet, G. R. Dvali, G. Gabadadze and A. I. Vainshtein, Phys. Rev. D 65 (2002) 044026.

[8] N. Arkani-Hamed, H. Georgi and M. D. Schwartz, Annals Phys. 305 (2003) 96.

[9] M. A. Luty, M. Porrati and R. Rattazzi, JHEP 0309 (2003) 029.

[10] V. A. Rubakov, arXiv:hep-th/0303125

[11] G. Gabadadze and M. Shifman, Phys. Rev. D 69 (2004) 124032; G. Gabadadze, Phys. Rev. D 70 (2004) 064005.

[12] A. Nicolis and R. Rattazzi, JHEP 0406 (2004) 059.

[13] A. Gruzinov, arXiv:astro-ph/0112246 G. Gabadadze and A. Iglesias, arXiv:hep-th/0407049

[14] A. Lue and G. Starkman, Phys. Rev. D 67 (2003) 064002; T. Tanaka, Phys. Rev. D 69 (2004) 024001; B. Kyae, JHEP 0403 (2004) 038.

[15] N. Kaloper, arXiv:hep-th/0501028

[16] F. A. E. Pirani, Proc. R. Soc. A 252 (1959) 96.

[17] P. C. Aichelburg and R. U. Sexl, Gen. Rel. Grav. 2 (1971) 303.
[18] T. Dray and G. 't Hooft, Nucl. Phys. B 253 (1985) 173; Class. Quant. Grav. 3 (1986) 825.

[19] V. Ferrari, P. Pendenza and G. Veneziano, Gen. Rel. Grav. 20 (1988) 1185.

[20] H. de Vega and N. Sanchez, Nucl. Ph. B 317 (1989) 706.

[21] C. Barrabes and P. A. Hogan, Phys. Rev. D 64 (2001) 044022.

[22] M. Hotta and M. Tanaka, Class. Q. Grav. 10 (1993) 307.

[23] J. Podolsky and J. Griffiths, Phys. Rev. D 56 (1997) 4756 .

[24] K. Sfetsos, Nucl. Phys. B 436 (1995) 721.

[25] N. Kaloper, unpublished (2000); C. Deffayet, Phys. Lett. B 502 (2001) 199.

[26] H. A. Chamblin and H. S. Reall, Nucl. Phys. B 562 (1999) 133.

[27] H. Collins and B. Holdom, Phys. Rev. D 62 (2000) 105009; Y. V. Shtanov, arXiv:hep-th/0005193

[28] N. Kaloper and A. D. Linde, Phys. Rev. D 59 (1999) 101303.

[29] A. Vilenkin, Phys. Lett. B 133 (1983) 177; J. Ipser and P. Sikivie, Phys. Rev. D 30 (1984) 712.

[30] E. Witten, Nucl. Phys. B 195 (1982) 481.

[31] R. Gregory, JHEP 0306 (2003) 041.

[32] N. Kaloper, Phys. Rev. D 60 (1999) 123506.

[33] G. R. Dvali, G. Gabadadze, M. Kolanovic and F. Nitti, Phys. Rev. D 64 (2001) 084004; Phys. Rev. D 65 (2002) 024031; C. Deffayet, G. R. Dvali and G. Gabadadze, Phys. Rev. D 65 (2002) 044023.

[34] I. Y. Aref'eva, Part. Nucl. 31 (2000) 169.

[35] R. Emparan, Phys. Rev. D 64 (2001) 024025.

[36] N. Arkani-Hamed, S. Dimopoulos, G. R. Dvali and N. Kaloper, Phys. Rev. Lett. 84 (2000) 586; N. Kaloper, JHEP 0405 (2004) 061.

[37] I. S. Gradshtein and I. M. Ryzhik, Table of Integrals, 
Series and Products, Academic Press, New York, 1980.

[38] A. Chamblin and G. W. Gibbons, Phys. Rev. Lett. 84 (2000) 1090.

[39] L. Randall and R. Sundrum, Phys. Rev. Lett. 83 (1999) 4690.

[40] G. 't Hooft, Phys. Lett. B 198 (1987) 61.

[41] I. J. Muzinich and M. Soldate, Phys. Rev. D 37 (1988) 359.

[42] D. Amati, M. Ciafaloni and G. Veneziano, Phys. Lett. B 197 (1987) 81; Int. J. Mod. Phys. A 3 (1988) 1615; Phys. Lett. B 216 (1989) 41; Nucl. Phys. B 347 (1990) 550.

[43] H. Verlinde and E. Verlinde, Nucl. Phys. B 371 (1992) 246.

[44] S. Kar and J. Maharana, Int. J. Mod. Phys. A 10 (1995) 2733; G. Arcioni, S. de Haro and M. O'Loughlin, JHEP
0107 (2001) 035.

[45] P. D. D'Eath and P. N. Payne, Phys. Rev. D 46 (1992) 658; Phys. Rev. D 46 (1992) 675; Phys. Rev. D 46 (1992) 694 .

[46] T. Banks and W. Fischler, arXiv:hep-th/9906038

[47] S. Dimopoulos and G. Landsberg, Phys. Rev. Lett. 87 (2001) 161602.

[48] S. B. Giddings and S. Thomas, Phys. Rev. D 65 (2002) 056010.

[49] G. F. Giudice, R. Rattazzi and J. D. Wells, Nucl. Phys. B 630 (2002) 293.

[50] D. M. Eardley and S. B. Giddings, Phys. Rev. D 66 (2002) 044011. 